\title{
Comparison of methods for the microbiological identification and typing of Cronobacter species in infant formula
}

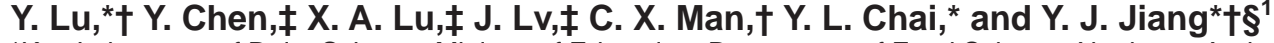 \\ *Key Laboratory of Dairy Science, Ministry of Education, Department of Food Science, Northeast Agricultural University, Harbin 150030, China \\ †National Dairy Engineering and Technical Research Center, Harbin 150086, China \\ ¥Chinese Academy of Inspection and Quarantine, Beijing 100123, China \\ §Synergetic Innovation Center of Food Safety and Nutrition, Harbin 150030, China
}

\begin{abstract}
Cronobacter species represent an emerging opportunistic foodborne pathogen associated with meningitis and necrotizing enterocolitis in infants. Current evidence indicates that powdered infant formula (PIF) is the main source of Cronobacter contamination. A total of 75 strains of Cronobacter spp. from different geographic regions, as well as from PIF processing environments, were identified and typed with different methods, including biochemical profiling by the API 20E system (bioMérieux, Marcy l'Etoile, France), protein profiling by matrix-assisted laser desorption/ionization time-of-flight mass spectrometry (MALDI-TOF MS), and genotypic profiling by ribotype. Analysis by MALDI-TOF MS and biochemical identification was more accurate compared with ribotype analysis. However, MALDI-TOF MS typing and ribotype analysis showed more discriminatory ability compared with biochemical phenotyping. In conclusion, MALDI-TOF MS is a rapid and reliable tool to identify Cronobacter spp. in PIF and has the potential to trace dissemination of Chronobacter along the production chain.

Key words: Cronobacter species, powdered infant formula, matrix-assisted laser desorption/ionization time-of-flight mass spectrometry (MALDI-TOF MS), ribotype
\end{abstract}

\section{INTRODUCTION}

Cronobacter is a newly emerging, gram-negative, motile, non-spore-forming, and peritrichous rod-shaped opportunistic foodborne pathogen (Lai, 2001). The organism can be detected in various types of foods [powdered infant formula (PIF), dairy milk, cheese products, meat, and vegetables), food production environments, and households (Iversen and Forsythe, 2004; Forsythe, 2005; Gurtler et al., 2005). Cronobacter infections in

Received June 15, 2013.

Accepted October 6, 2013.

${ }^{1}$ Corresponding author: yujun_jiang@163.com
PIF for neonates and infants have received attention, with sporadic and outbreak cases reported globally (Block et al., 2002; Himelright et al., 2002). Infection with Cronobacter is associated with meningitis and necrotizing enterocolitis in infants (Caubilla-Barron et al., 2007; Hartmann et al., 2010).Current evidence indicates that PIF is the main medium of Cronobacter spp. dissemination (van Acker et al., 2001; Javůrková et al., 2012). To date, there are no reports of Cronobacter spp. infecting infants in China.

The definition of the Cronobacter genus was revised in 2012. The species Enterobacter sakazakii was formally replaced with 7 species of the Cronobacter genus: Cronobacter sakazakii, Cronobacter malonaticus, Cronobacter muytjensii, Cronobacter turicensis, Cronobacter dublinensis, Cronobacter universalis, and Cronobacter condimenti (Iversen et al., 2008; Joseph et al., 2012). The isolates examined in this study were all C. sakazakii strains.

For this study, 75 C. sakazakii strains were isolated from samples deposited in the National Dairy Testing Center (Harbin, China) from 2009 to 2012 and from a wet-mixing PIF-manufacturing facility over a 10 -mo period, including samples from raw materials to final products in the factory environment (Mullane et al., 2007; Table 1). Several microbiologic identification methods have been used to identify Cronobacter spp. isolates (Caubilla-Barron et al., 2007). The isolates in the current study were identified using the API20E system (bioMérieux, Marcy l'Etoile, France), and classification was based on different biochemical characters. The present study evaluated the application of matrixassisted laser desorption ionization-time of flight mass spectrometry (MALDI-TOF MS) for identification and protein profiling of C. sakazakii strains (Karamonová et al., 2013). With the rapid development of bacterial analysis by MALDI-TOF MS, through singlecomponent and multi-component determination in the group, the identification and classification of bacteria was performed on the basis of the acquired mass spectra matched to the MALDI-TOF database (Lay, 2000). Barbuddhe et al. (2008) were able to separate 
Table 1. Analyzed bacterial strains and isolates from the commercially available powdered infant formulas and raw materials and their production facilities in China

\begin{tabular}{|c|c|c|}
\hline Bacterial strain & $\begin{array}{l}\text { No. of } \\
\text { strains }\end{array}$ & $\begin{array}{l}\text { Strain }(s) \text { or } \\
\text { source }\end{array}$ \\
\hline Cronobacter spp. & 5 & $\begin{array}{l}\text { ATCC }^{1} 29544 \\
\text { ATCC } 51329 \\
\text { ATCC } 12868 \\
\text { ATCC } 29004 \\
\text { ATCC BAA-894 }\end{array}$ \\
\hline ES60, ES65, ES66 & 3 & Environment \\
\hline ES30, ES31, ES32, ES61, ES63, ES64, ES68, ES70, ES71, ES72, ES73, ES75, ES76, ES77, ES78 & 15 & Final products \\
\hline ES11, ES12, ES13, ES14, ES15, ES16, ES17, ES18, ES19, ES20, ES22, ES23 & 12 & Milk powders \\
\hline $\begin{array}{l}\text { ES1, ES2, ES3, ES4, ES8, ES9, ES10, ES21, ES24, ES25, ES26, ES27, ES28, ES29, ES34, ES35, } \\
\text { ES41, ES43, ES44, ES47, ES48, ES49, ES50, ES51, ES52, ES53, ES54, ES55, ES56, ES69 }\end{array}$ & 30 & Powdered infant formula \\
\hline
\end{tabular}

Listeria monocytogenes isolates to the subtype level. In their study, the pulsed-field gel electrophoresis lineages were in complete agreement with the MALDI-based groupings. The RiboPrinter Microbial Characterization System (DuPont/Qualicon Inc., Wilmington, DE) has also been used to analyze specific genetic fingerprints and to type C. sakazakii strains (Gaston, 1988; Poilane et al., 1993).

The objective of this study was to identify and type Cronobacter isolates from PIF and related production environment. Several different methods, including biochemical identification, mass spectrometry, and ribosome analysis, were used to identify and characterize these strains and the results compared. The advantages and disadvantages of these methods and the relationship between them were analyzed to find a more powerful method to reduce Cronobacter contamination during dairy production and to trace the contamination source along the processing chain.

\section{MATERIALS AND METHODS}

\section{Detection and Isolation of Bacterial Strains}

Cronobacter spp. isolates in this study were detected based on GB4789.40-2010 (Ministry of Health of the People's Republic of China, 2010), the national food safety standard method for food microbiological examination used in China. Cronobacter sakazakii strains were isolated from commercially available PIF and their production factories in China, and none was associated with confirmed clinical cases. Specifically, C. sakazakii was isolated in PIF production facilities in powder samples obtained from the drying tower, raw materials for PIF production, final PIF products, waste powder lumps in the dryer, swabs taken outside the facility drying tower, an operator's hands, carriers' wheels, vacuum, ground leakage, powder miller, bag-filling platform, and packing materials in the factory environment. The strains used are summarized in Table 1.

\section{Reference Strains}

The biochemical characteristics of $C$. sakazakii strain ATCC 29544 were used as the standard biotype to standardize the sample preparation protocol for MALDI-TOF MS analysis. Cronobacter muytjensii strain ATCC 51329 was used as a reference strain to ensure reproducibility between ribotype experiments.

\section{Bacterial Growth}

To standardize the analysis protocol, several experimental parameters, including culture medium and culture time (Smole et al., 2002), were evaluated. Nutrient agar, tryptic soy agar (TSA), violet red bile agar, and C. sakazakii chromogenic medium were tested to determine the influence of different medium on strain identification (Lynn et al., 1999; Madonna et al., 2000). Extended culture periods of $C$. sakazakii on agar at $36^{\circ} \mathrm{C}$ for 18,24 , and $48 \mathrm{~h}$ were evaluated to select the optimal culture time.

\section{Biochemical Identification}

The API20E system (bioMérieux), consisting of a strip of 20 individual, miniaturized plastic test tubes (cupules) and each containing different reagents, was used according to the manufacturer's instructions. This system determines the metabolic capabilities and allows for the identification of the genus and species of enteric bacteria in the family Enterobacteriaceae. Appropriate positive and negative controls were included. 


\section{MALDI-TOF MS}

A MALDI-TOF mass spectrometer (Autoflex II and Microflex, Bruker Daltonics, Bremen, Germany) and BioNumerics database and analysis software (Applied Maths, Sint-Martens-Latem, Belgium) were used in this study. A dendrogram was created using the DICE coefficient and unweighted pair group method with arithmetic mean (UPGMA).

All mass spectra were acquired under a linear, delayed-extraction, positive-ion mode $\left(\mathrm{N}_{2}, 337 \mathrm{~nm}\right)$ and the frequency was intercalated at $30 \mathrm{~Hz}$ within a mass range from 2,000 to 20,000 Da (Barbuddhe et al., 2008). The acceleration voltage was $20 \mathrm{kV}$ and the extraction delay voltage was maintained at $18.250 \mathrm{kV}$; the delay time was 10 ns. Each sample was acquired and analyzed for 20,000 laser shots $(10 \times 2,000 \mathrm{~Hz}$ shots from different positions toward the target). The calibration material was a mixture of protein and peptide (Bruker Daltonics). The calibration process can minimize the error of the experiment to $<5$ Da. All mass values were reported as average masses.

\section{Preparation of Bacterial Cells for MALDI-TOF MS}

Approximately $5 \mathrm{mg}$ of bacteria was suspended in $300 \mu \mathrm{L}$ of double-distilled water in a 1.5-mL Eppendorf tube. Then, $900 \mu \mathrm{L}$ of ethanol was added and the solution was mixed by vortexing thoroughly. The sample in the ethanol-water solution was centrifuged at 9,180 $\times g$ for 2 min to collect the cells. The supernatant was removed and the cells were air dried. The sample was then prepared as described by Barbuddhe et al. (2008). Briefly, $50 \mu \mathrm{L}$ of formic acid (70\% in water, $\mathrm{vol} / \mathrm{vol}$ ) was added to the bacterial pellet and mixed thoroughly; then, $50 \mu \mathrm{L}$ of acetonitrile was added. After centrifugation at $9,180 \times g$ for $2 \mathrm{~min}, 1 \mu \mathrm{L}$ of supernatant containing the bacterial extract was placed onto a spot on the steel target plate and air-dried at room temperature for $2 \mathrm{~min}$. Subsequently, the sample was overlaid with $2 \mu \mathrm{L}$ of MALDI matrix (a saturated solution of $\alpha$-cyano-4-hydroxy-cinnamic acid in 50\% acetonitrile- $2.5 \%$ trifluoroacetic acid, stored at $4^{\circ} \mathrm{C}$ ) and dried again. Subsequently, the same method was applied to rest of the samples.

\section{MALDI-TOF MS Data Analysis}

The Flexcontrol software (Bruker Daltonics) was used to collect related data, which generated lists of most significant peaks of the spectra. The Biotyper 2.0 software (Bruker Daltonics) was used to identify objective bacteria and to cluster strains according to their genetic relationship. The Flexanalysis software was used to analyze the mass peak information. The whole procedure from processing to identification was performed automatically. The list of specific peaks generated by this program constituted the bacterial molecular profiles, and species-specific biomarkers were determined by comparing the obtained peak lists (Stephan et al., 2010).

\section{Ribotype}

Ribotyping was performed using the automated RiboPrinter Microbial Characterization System (DuPont/ Qualicon Inc., Wilmington, DE) to produce genetic fingerprints, called riboprint or ribotyping patterns. The strains were grown on TSA at $36^{\circ} \mathrm{C}$ for $24 \mathrm{~h}$ to obtain pure isolated colonies. Then, a colony pick was touched to the solid lawn and placed into a tube, which was transferred into $200 \mu \mathrm{L}$ of sample buffer (supplied in the DuPont kit) before being mixed by vortexing for $5 \mathrm{~s}$. After ensuring that all cells transferred, the pick was discarded. According to standard procedures (Bruce, 1996), bacterial cells were lysed, DNA fragments were generated with EcoRI, and the fragments were hybridized with a labeled ribosomal RNA operon. The hybridization patterns were observed by chemiluminescence and recorded with a charge-coupled device camera. Algorithms for data normalization were used to obtain ribotyping patterns that were downloaded to Bionumerics v5.01 (Applied Maths), and the UPGMA algorithm was constructed using a Dice coefficient under optimization of $1.0 \%$ and a position tolerance of $1.5 \%$. The genetic diversity and relatedness of $C$. sakazakii strains were compared at $90 \%$ similarity.

\section{RESULTS}

\section{Biochemical Identification}

The biochemical identification of Cronobacter spp. isolates in this study was performed using an API20E gallery (bioMérieux). All isolates were identified as $C$. sakazakii except for isolate ES59, which did not present an acceptable identification result via API20E. In this assay, $98.7 \%$ of the strains tested resulted in good identification.

According to the identification results by the API20E system, the $o$-nitrophenyl- $\beta$-D-galactoside, sodium citrate, glucose, mannitol, rhamnose, saccharose, melibiose, amygdalin, and arabinose reactions were all positive, and the lysine decarboxylase, tryptophan decarboxylase, hydrogen sulfide, and oxidase reactions were all negative for C. sakazakii. These strains were divided into 8 biotypes according to a combination of 6 different results of API20E (Table 2). The biochemical character 
of type strain ATCC 29544 was designated as biotype 1 (3305373), on which all comparisons were made, and different biochemical characters were defined as new biotypes. Biotype 1 contained 31 isolates $(41.9 \%)$, biotype 2 had 19 isolates (25.7\%), and biotype 3 contained 12 isolates (16.2\%). Isolate ES59 was not identified because an acceptable result was not obtained in the API 20E system. The bioMérieux database does not recognize the Cronobacter genus, and therefore further identification to the species level was not possible.

\section{Standardization of Sample Preparation for MALDI-TOF MS Analysis}

The differences in the peaks from the mass spectrometry analysis were not obvious when the preparation of these strains was done on 4 media (nutrient agar, TSA, violet red bile agar, and C. sakazakii chromogenic medium) and using 3 different cultivation times (18, 24 , and $48 \mathrm{~h}$ ) in strain identification. Therefore, the optimal protocol was set as follows: incubate the bacteria on TSA at $36^{\circ} \mathrm{C}$ for $24 \mathrm{~h}$. Sample preparation and mass spectrometric parameters were evaluated first to provide good reproducibility and accuracy of the measured data. Preliminary experiments were performed using strain C. sakazakii ATCC 29544.

\section{Identification and Typing by MALDI-TOF MS}

All tested strains were identified as $C$. sakazakii by MALDI-TOF MS, including ES59. Strains with log score values of 2.0 or greater accounted for $92.0 \%$ of strains. For Cronobacter spp., the peaks near $m / z 4,738$, $m / z 5,380$, and $m / z 5,742$ may be used as reference biomarkers under the present experimental conditions (Figure 1).

The distance level (between 0 and 1,000) between strains represents the genetic relationship among strains. A difference of 0 between strains indicates an identical mass spectrogram belonging to the same category, whereas a difference of 1,000 shows 2 strains with the farthest genetic relationship within the existing clustering results. All C. sakazakii strains in this study were classified into clusters based on distance levels; that is, 7 clusters by MALDI-TOF MS at a distance level of 600,11 clusters at a distance level of 400 , 16 clusters at a distance level of 300 , and 23 clusters at a distance level of 200 . The 75 strains could be grouped into 36 clusters with 1 to 14 isolates in each cluster at a distance level of 100 . Cluster 36, containing 13 isolates, was the predominant bacterial type and accounted for $17.3 \%$ of the strains. Strains ATCC BAA-894 and ATCC 29544 had almost the same mass spectrogram and were close to ATCC 12868; these strains showed a lesser genetic relationship with ATCC 51329 (Figure 2 ). These results are consistent with previous classifications based on genetic characteristics (Iversen et al., 2008).

\section{Identification and Typing of C. sakazakii Strains by Riboprinter}

Genus and species are automatically generated by comparing the Riboprinter pattern to an identification database of about 7,000 patterns covering more than 1,400 species, integrating data from multiple Riboprinter systems into a central database for better analysis. Among the tested strains, $89.3 \%$ were identified as the target bacteria, and these 75 strains were classified into 18 ribotypes. A dendrogram was constructed using a Dice relative coefficient and Bionumerics software; approximately 10 bands were produced in a restriction digest with lengths ranging from 1 to $50 \mathrm{~kb}$. Analysis of the results revealed multiple digestion patterns as follows: 1 pattern included 13 strains, 1 pattern included 14 strains, 1 pattern included 20 strains; and 9 strains showed unique ribotype patterns. Strains ATCC 51329 and ATCC BAA894 showed approximately $90 \%$ similarity in ribotype 6, whereas ATCC 29004 and ATCC 12868 exhibited approximately $90 \%$ similarity in ribotype 15 ; ATCC 29544 displayed a unique ribotype.

Table 2. Results of biochemical reactions for 8 biotypes of Cronobacter

\begin{tabular}{lcccccccc}
\hline & \multicolumn{8}{c}{ Biotype $^{2}$} \\
\cline { 2 - 9 } Assimilation $_{\text {substrate }^{1}}$ & 3305373 & 3305773 & 3305173 & 1305373 & 3315373 & 3205173 & 3304373 & 3205373 \\
\hline ADH & + & + & + & - & + & + & + & + \\
ODC & + & + & + & + & + & - & + & - \\
URE & - & - & - & - & + & - & - & - \\
VP & + & + & + & + & + & + & - & + \\
INO & + & + & - & + & + & - & + & + \\
SOR & - & + & - & - & - & - & - & -
\end{tabular}

${ }^{1} \mathrm{ADH}=$ arginine dihydrolase, $\mathrm{ODC}=$ ornithine decarboxylase, $\mathrm{URE}=$ urease, $\mathrm{VP}=$ Voges and Proskauer, $\mathrm{INO}=$ inositol, and $\mathrm{SOR}=$ sorbitol.

${ }^{2}$ Biotypes represent clusters of strains having the same combination of biochemical reaction results. 


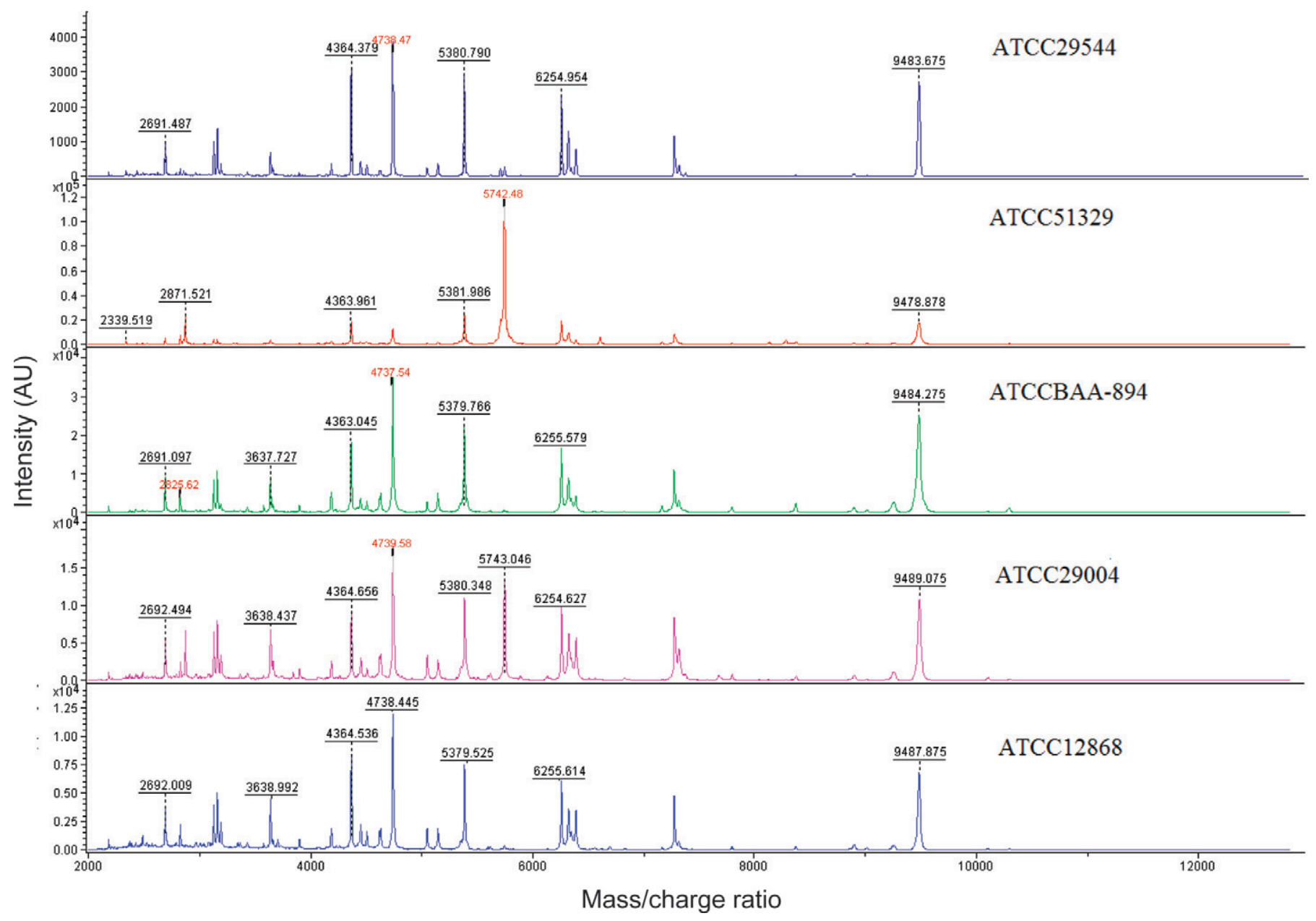

Figure 1. Matrix-assisted laser desorption/ionization time-of-flight mass spectrometry (MALDI-TOF MS) spectra of 5 Cronobacter spp. reference strains. The mass intensity is on the $\mathrm{y}$-axis, and the $\mathrm{x}$-axis indicates the mass-to-charge ratio $(\mathrm{m} / z)$. For Cronobacter spp., the peaks near $m / z 4,364, m / z 5,380$, and $m / z 9,484$ are genus peaks; the peaks near $m / z 4,738$ and $m / z 5,742$ are species-specific peaks. ATCC $=$ American Type Culture Collection. Color version available in the online PDF.

Strains ES51, ES52, and ES53, collected from the same region (Shanxi, China), had the same profile, and strains ES17, ES18, ES22, and ES23 from another region (Shandong, China) presented with another identical profile. Ten C. sakazakii strains isolated from the final products, powder lumps, and the environment of one dry milk factory in different years $(2009,2011$, and 2012) were identified with same ribotype. Eight isolates (ES31, ES32, ES62, ES68, ES75, ES76, ES77, and ES78) obtained from 4 different batches in the same factory also showed the same ribotype profile (Figure 3).

\section{Comparison of Clustering Classification of the 75 Strains by 3 Methods}

Bacteria in the same MALDI-TOF MS cluster contained a variety of biochemical phenotypes; for example, MALDI-TOF MS type 36 showed 4 biochemical phenotypes (biotype 1-4), and MALDI-TOF MS type 34 exhibited 3 biochemical phenotypes (biotypes 1, 3, and 4). The same biochemical phenotype was observed in different MALDI-TOF MS clusters. We observed no one-to-one correspondence between the 2 typing methods.

Bacteria in the same MALDI-TOF MS type also contained a variety of ribotypes; for example, MALDITOF MS type 36 showed 6 ribotypes (ribotypes 4, 6 , $8,9,15$, and 17) and MS type 34 contained 5 ribotypes (ribotype 6, 8, 9, 14, and 15), again indicating the lack of one-to-one correspondence between the 2 typing methods.

Each ribotype contained more than 2 biotypes. Classifications based on mass spectrometry and biochemical phenotypic profiles showed no direct correspondence except that all 5 reference strains were clustered into biotype 1 . 


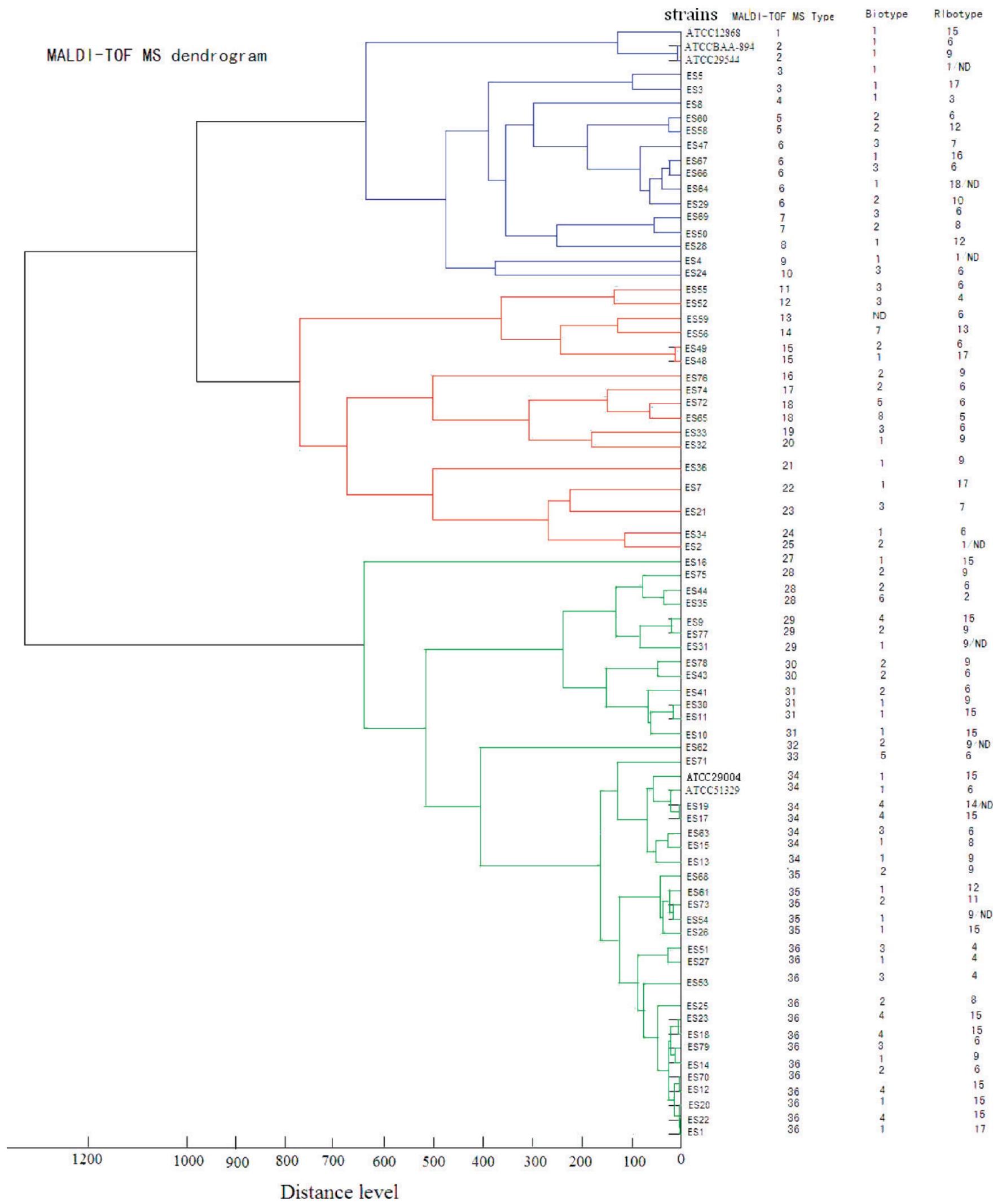

Figure 2. Clustering of 75 Cronobacter sakazakii strains by matrix-assisted laser desorption/ionization time-of-flight mass spectrometry (MALDI-TOF MS), biochemical test, and ribotype. Note: dendrogram is based on MALDI-TOF. ND = not determined. Source of ES strain numbers given in Table 1. ATCC = American Type Culture Collection. Color version available in the online PDF. 
Strains ES12, ES18, ES22, and ES23 isolated from milk powder were all classified into MALDI-TOF MS type 36, ribotype 15, and biotype 4. Strains ES18, ES22, and ES23 were isolated in Shandong. These were the only strains showing highly consistent classification with the 3 classification methods in this study (Figure 2).

\section{DISCUSSION}

Biochemical identification is widely used for bacterial detection. It is easy and simple to handle, but phenotypic analysis has limited value for investigative studies due to the subjective nature of the results. Disadvantages of biochemical testing are that it is laborious, and time consuming, with poor discriminatory power (Cetinkaya et al., 2013), and it is limited to distinguishing strains within a given species (Bingen et al., 1994). In the current study, $98.6 \%$ of 75 C. sakazakii strains were Voges and Proskauer-positive and 20\% were inositol-negative, which was consistent with the conclusion of Farmer et al. (1980), who found that 98\% of 57 E. sakazakii strains were Voges and Proskauerpositive and 25\% were inositol-negative. Although ES59 (API20E code: 7315773 ) was repeatedly not identified as $C$. sakazakii in this study using biochemical testing, this strain was classified as C. sakazakii by both RiboPrinter and MALDI-TOF MS; $16 \mathrm{~S}$ rDNA gene sequencing of ES59 confirmed this classification (Table 3). A few strains that are damaged in the environment (such as by drying, radiation, and so on) might contain metabolic anomalies and thus may not perform well in some biochemical characterizations, which might have been the case for ES59. To avoid the potential risk of false-negative results from strains obtained from raw materials used in production, a more accurate and fast method of $C$. sakazakii identification is needed to supplement existing detection methods.

Reproducibility, speed, automation, and high throughput are the major advantages of MALDI-TOF MS method, which takes less than 2 min to detect each sample spot (Bright et al., 2002). In total, 75 strains were correctly detected as $C$. sakazakii, which was concordant with the biochemical methods. The MALDITOF MS method had a stronger typing ability compared with biochemical phenotyping and ribotyping in this study, indicating that mass spectra have good classification ability in clustering C. sakazakii. Strains ES65, ES60, and ES66 were isolated from a bag-filling platform, a star-shaped valve, and a U-shaped valve of the processing equipment, respectively; ES24 was collected from the final products in the same factory. These 4 strains showed very similar mass spectra and classification results (Figure 4). Strains ES60, ES66,

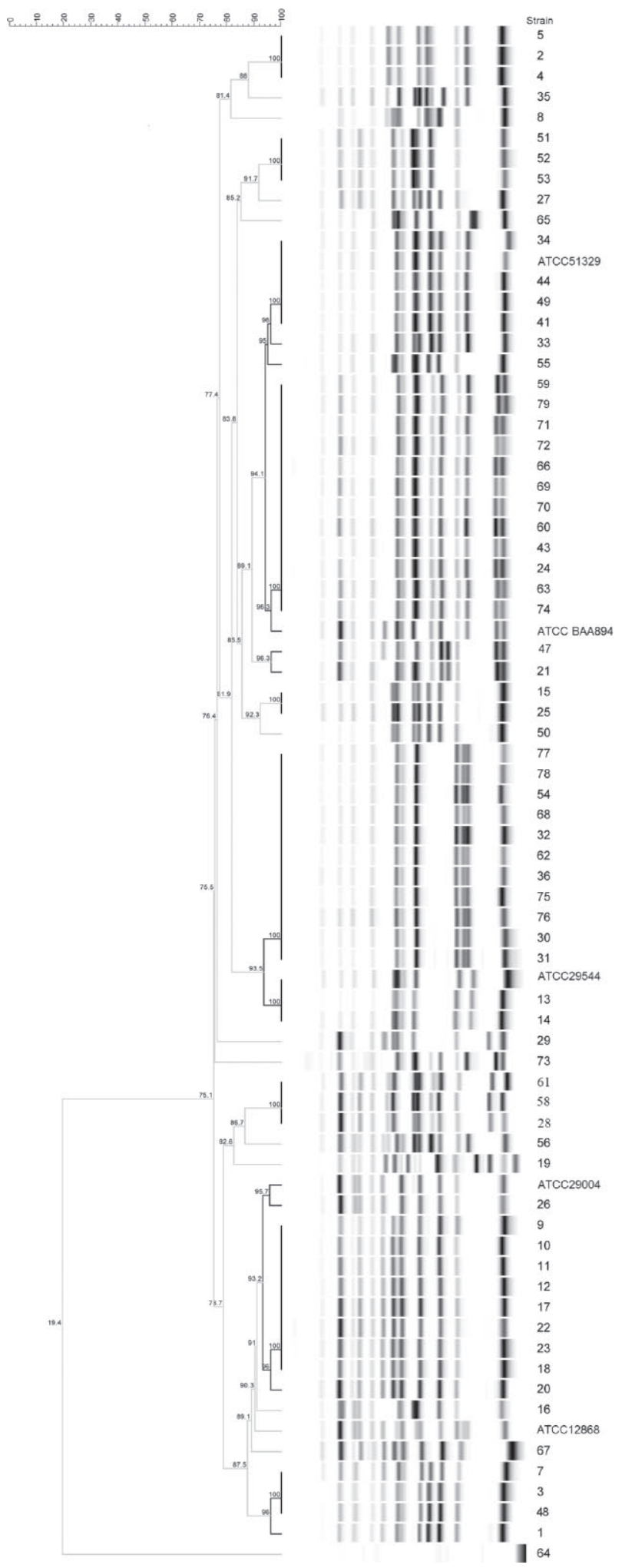

Figure 3. Ribotype dendrogram of 75 Cronobacter sakazakii strains. A Dice coefficient and an unweighted pair group method with arithmetic mean (UPGMA) algorithm were used with an optimization of $1.0 \%$ and a position tolerance of $1.5 \%$. The scale bar represents the percentage similarity. The strain numbers represent the ES isolate numbers; ATCC $=$ American Type Culture Collection. 
Table 3. Identification of 9 strains of Cronobacter by different methods ${ }^{1}$

\begin{tabular}{lllll}
\hline Strain & Biochemical & MALDI-TOF MS & RiboPrinter & 16S rDNA (NCBI) \\
\hline ES2 & C. sakazakii & C. sakazakii & Citrobacter freundii & HQ880299.1 \\
ES4 & C. sakazakii & C. sakazakii & Cit. freundii & GU227670.1 \\
ES5 & C. sakazakii & C. sakazakii & Cit. freundii & HQ880299.1 \\
ES19 & C. sakazakii & C. sakazakii & Proteus mirabilis & EF059825.1 \\
ES31 & C. sakazakii & C. sakazakii & Facklamia ignava & HQ880317.1 \\
ES54 & C. sakazakii & C. sakazakii & Providencia stuartii & HQ880317.1 \\
ES59 & Unidentified & C. sakazakii & C. sakazakii & HQ880296.1 \\
ES62 & C. sakazakii & C. sakazakii & Facklamia ignava & HQ880317.1 \\
ES64 & C. sakazakii & C. sakazakii & Hydrogenophaga flava & GU227695.1 \\
\hline
\end{tabular}

${ }^{1}$ Biochemcal = identification using API20E system (bioMérieux, Marcy l'Etoile, France) for identifying Enterobacteriaceae; MALDI-TOF MS = identification by matrix-assisted laser desorption/ionization time-oflight mass spectrometry; RiboPrinter = identification by automated RiboPrinter Microbial Characterization System (DuPont/Qualicon Inc., Wilmington, DE); 16S rDNA (NCBI) = identification by genomic sequencing of $16 \mathrm{~S}$ rDNA, with National Center for Biotechnology Information locus identifier.

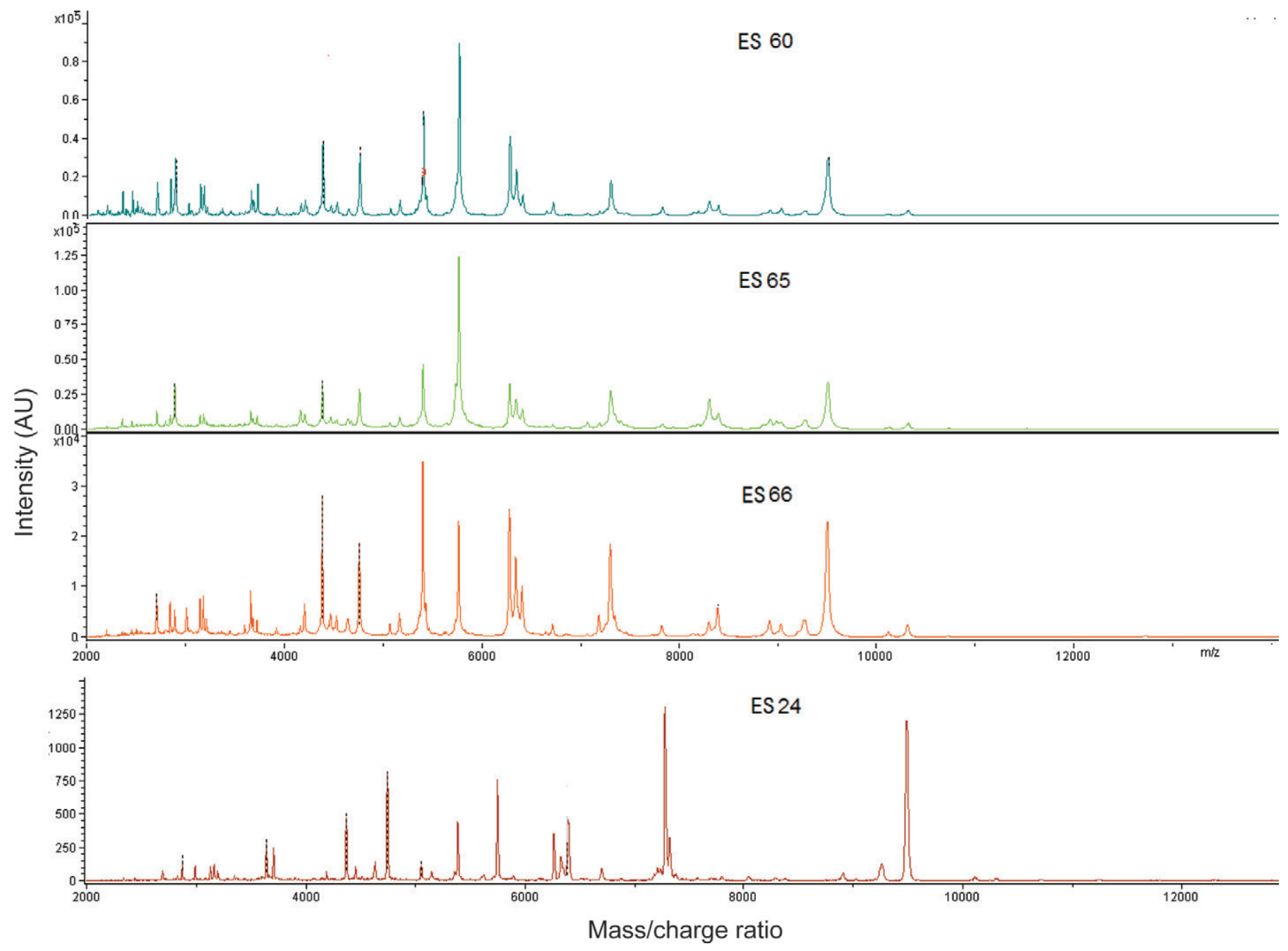

Figure 4. Matrix-assisted laser desorption/ionization time-of-flight mass spectrometry (MALDI-TOF MS) spectra of environmental strains isolated from platform (ES65), star-shaped valve (ES60), and U-shaped valve (ES66); ES24 is the reference sample from the same factory. The mass intensity is shown on the $\mathrm{y}$-axis, and the $\mathrm{x}$-axis indicates the mass-to-charge ratio $(\mathrm{m} / \mathrm{z})$. Color version available in the online PDF. 
and ES24 belonged to ribotype 6 and were very similar to ES65 (Figure 3). The recovery of C. sakazakii from multiple sites in the processing facility suggests that final product contamination occurs throughout the environment of the processing facility. These results provide an easy way to determine which C. sakazakii strain was isolated from an indicated site in the factory, in agreement with the findings of Mullane et al. (2007), who suggested that the manufacturing environment serves as a key route for the sporadic contamination of PIF.

The accuracy of ribosome detection in the current study was is inferior to that of mass spectrogram and biochemical identification. Strains ES2, ES4, ES5, ES19, ES31, ES54, ES62, and ES64 were identified as bacterial strains other than C. sakazakii by Riboprinter in this study, which did not match the data from biochemical identification or mass spectrometry. Among them, 8 strains were reanalyzed by $16 \mathrm{~S}$ rDNA gene sequencing, and the results indicated that they were all C. sakazakii (Table 3). Compared with MALDI-TOF MS, ribotyping of some isolates showed regional or temporal continuity [i.e., strains from the same area had similar ribotypes (regional continuity) or the strains isolated in the same area in different years came from the same contamination points, which had been previously overlooked (temporal continuity)], and thus ribotyping exhibited an advantage in that isolates from the same region displayed a high degree of similarity and, therefore, contamination points could be traced during the production process. Ten isolates from different dairy products in one factory in different years showed the same ribotype in this study, suggesting a sole source of contamination in the production environment.

Strains identical by all 3 methods were rare among the 75 strains tested in this study (Figure 2), perhaps because the proteins analyzed by mass spectrometry are on the membrane, (e.g., cell receptors, adhesins) and thus prone to laser desorption, whereas biochemical identification utilized traditional biochemistry and fermentation reactions to identify Enterobacteriaceae. Ribotyping involves enzyme digestion of the whole genome. Theoretically, no direct correspondence should exist among the 3 detection and typing methods.

In this study, phenotypic methods, protein profile, and genotypic methods for the identification and typing of Cronobacter isolates were performed. Analysis by MALDI-TOF MS and biochemical identification showed better identification accuracy compared with Riboprinter analysis, whereas MALDI-TOF MS and ribotype analysis showed more discrimination between strains compared with biochemical phenotyping. Use of both methods (MALDI-TOF plus ribotyping) together had the potential for rapid analysis and the ability to detect slight alterations between phylogenetically similar strains. Understanding the contamination potential and trend of the pathogen is helpful to prevent and control the contamination of the final product by C. sakazakii during infant formula production (FAO/ WHO, 2008), which is an important first step to reduce the risk of PIF contamination and to trace the pathogenic bacteria.

\section{ACKNOWLEDGMENTS}

We are grateful to the Beijing Academy of Science and Technology for Inspection and Quarantine (Beijing, China) for providing the matrix-assisted laser desorption/ionization time-of-flight mass spectrometer and reference strains used in this study. This project was supported by grant from the National Key Technology Support Program (No. 2012BAK17B04).

\section{REFERENCES}

Barbuddhe, S. B., T. Maier, G. Schwarz, M. Kostrzewa, H. Hof, E. Domann, T. Chakraborty, and T. Hain. 2008. Rapid identification and typing of Listeria species by matrix-assisted laser desorption ionization-time of flight mass spectrometry. Appl. Environ. Microbiol. 74:5402-5407.

Bingen, E. H., E. Denamur, and J. Elion. 1994. Use of ribotyping in epidemiological surveillance of nosocomial outbreaks. Clin. Microbiol. Rev. 7:311-327.

Block, C., O. Peleg, N. Minster, B. Bar-Oz, A. Simhon, I. Arad, and M. Shapiro. 2002. Cluster of neonatal infections in Jerusalem due to unusual biochemical variant of Enterobacter sakazakii. Eur. J. Clin. Microbiol. Infect. Dis. 21:613-616.

Bright, J. J., M. A. Claydon, M. Soufian, and D. B. Gordon. 2002. Rapid typing of bacteria using matrix-assisted laser desorption ionisation time-of-flight mass spectrometry and pattern recognition software. J. Med. Microbiol. 48:127-138.

Bruce, J. 1996. Automated system rapidly identifies and characterizes microorganisms in food. Food Technol. 50:77-81.

Caubilla-Barron, J., E. Hurrell, S. Townsend, P. Cheetham, C. LocCarrillo, O. Fayet, M.-F. Prere, and S. J. Forsythe. 2007. Genotypic and phenotypic analysis of Enterobacter sakazakii strains from an outbreak resulting in fatalities in a neonatal intensive care unit in France. J. Clin. Microbiol. 45:3979-3985.

Cetinkaya, E., S. Joseph, K. Ayhan, and S. J. Forsythe. 2013. Comparison of methods for the microbiological identification and profiling of Cronobacter species from ingredients used in the preparation of infant formula. Mol. Cell. Probes 27:60-64.

FAO/WHO. 2008. Enterobacter sakazakii (Cronobacter spp.) in powdered follow-up formula. Microbiological risk assessment series no. 15. Accessed Aug. 16, 2010. http://www.who.int/foodsafety/publications/micro/mra_followup/en/.

Farmer, J. J., M. A. Asbury, F. W. Hickman, D. J. Brenner, and Enterobacteriaceae Study Group. 1980. Enterobacter sakazakii: A new species of 'Enterobacteriaceae' isolated from clinical specimens. Int. J. Syst. Bacteriol. 30:569-584.

Forsythe, S. J. 2005. Enterobacter sakazakii and other bacteria in powdered infant milk formula. Matern. Child Nutr. 1:44-50.

Gaston, M. A. 1988. Enterobacter, an emerging nosocomial pathogen. J. Hosp. Infect. 11:197-208.

Gurtler, J. B., J. L. Kornacki, and L. Beuchat. 2005. Enterobacter sakazakii: A coliform of increased concern to infant health. Int. J. Food Microbiol. 104:1-34.

Hartmann, I., P. Carranza, A. Lehner, R. Stephan, L. Eberl, and K. Riedel. 2010. Genes involved in Cronobacter sakazakii biofilm formation. Appl. Environ. Microbiol. 76:2251-2261. 
Himelright, I., E. Harris, V. Lorch, and M. Anderson. 2002. Enterobacter sakazakii infections associated with the use of powdered infant formula-Tennessee, 2001. JAMA 287:2204-2205.

Iversen, C., and S. J. Forsythe. 2004. Isolation of Enterobacter sakazakii and other Enterobacteriaceae from powdered infant formula milk and related products. Food Technol. 21:771-777.

Iversen, C., N. Mullane, B. McCardell, B. D. Tall, A. Lehner, S. Fanning, R. Stephan, and H. Joosten. 2008. Cronobacter gen. nov., a new genus to accommodate the biogroups of Enterobacter sakazakii, and proposal of Cronobacter sakazakii gen. nov., comb. nov., Cronobacter malonaticus sp. nov., Cronobacter turicensis sp. nov., Cronobacter muytjensii sp. nov., Cronobacter dublinensis sp. nov., Cronobacter genomospecies 1, and of three subspecies, Cronobacter dublinensis ssp. dublinensis ssp. nov., Cronobacter dublinensis ssp. lausannensis ssp. nov. and Cronobacter dublinensis ssp. lactaridi ssp. nov. Int. J. Syst. Evol. Microbiol. 58:1442-1447.

Javůrková, B., M. Blažková, L. Fukal, and P. Rauch. 2012. Short communication: Rapid detection of genus Cronobacter in powdered infant formula milk. Eur. Food Res. Technol. 234:1099-1104.

Joseph, S., E. Cetinkaya, H. Drahovska, A. Levican, M. J. Figueras, and S. J. Forsythe. 2012. Int. J. Syst. Evol. Microbiol. 62:12771283.

Karamonová, L., P. Junková, D. Mihalová, B. Javůrková, L. Fukal, P. Rauch, and M. Blazková. 2013. The potential of matrix-assisted laser desorption/ionization time-of-flight mass spectrometry for the identification of biogroups of Cronobacter sakazakii. Rapid Commun. Mass Spectrom. 27:409-418.

Lai, K. K. 2001. Enterobacter sakazakii infections among neonates, infants, children, and adults. Medicine 80:113-122.

Lay, J. O., Jr. 2000. MALDI-TOF mass spectrometry and bacterial taxonomy. TrAC Trends Anal. Chem. 19:507-516.

Lynn, E. C., M. C. Chung, W. C. Tsai, and C. C. Han. 1999. Identification of Enterobacteriaceae bacteria by direct matrix-assisted laser desorption/ionization mass spectrometric analysis of whole cells. Rapid Commun. Mass Spectrom. 13:2022-2027.

Madonna, A. J., F. Basile, I. Ferrer, M. A. Meetani, J. C. Rees, and K. J. Voorhees. 2000. On-probe sample pretreatment for the detection of proteins above $15 \mathrm{kDa}$ from whole cell bacteria by matrix-assisted laser desorption ionization time-of-flight mass spectrometry. Rapid Commun. Mass Spectrom. 14:2220-2229.

Ministry of Health of the People's Republic of China. 2010. GB4789.402010. National food safety standard: Food microbiological examination: Enterobacter sakazakii[S]. China Standard Press, Beijing, China.

Mullane, N. R., P. Whyte, P. G. Wall, T. Quinn, and S. Fanning. 2007. Application of pulsed-field gel electrophoresis to characterize and trace the prevalence of Enterobacter sakazakii in an infant formula processing facility. Int. J. Food Microbiol. 116:73-81.

Poilane, I., P. Cruaud, E. Lachassinne, E. Grimont, P. A. D. Grimont, M. Collin, J. Gaudelus, J. C. Torlotin, and A. Collignon. 1993. Enterobacter cloacae cross-colonization in neonates demonstrated by ribotyping. Eur. J. Clin. Microbiol. Infect. Dis. 12:820-826.

Smole, S. C., L. A. King, P. E. Leopold, and R. D. Arbeit. 2002 Sample preparation of gram-positive bacteria for identification by matrix assisted laser desorption/ionization time-of-flight. J. Microbiol. Methods 48:107-115.

Stephan, R., D. Ziegler, V. Pflüger, G. Voge, and A. Lehner. 2010. Rapid genus- and species-specific identification of Cronobacter spp. by matrix-assisted laser desorption ionization-time of flight mass spectrometry. J. Clin. Microbiol. 48:2846-2851.

van Acker, J., F. de Smet, G. Muyldermans, A. Bougatef, A. Naessens, and S. Lauwers. 2001. Outbreak of necrotizing enterocolitis associated with Enterobacter sakazakii in powdered milk formula. J. Clin. Microbiol. 39:293-297. 